\title{
Análisis del tiempo de entrenamiento y su relación con los episodios de peritonitis
}

\author{
Ana Isabel Aguilera-Flórez, Aránzazu Sastre-López, Blanca Linares-Fano, Juan Ramón Guerra-Ordoñez, Ana \\ Cristina Alonso-Rojo, Mario Prieto-Velasco
}

Servicio de Nefrología. Complejo Asistencial Universitario de León. León. España

Como citar este artículo:

Aguilera-Flórez AI, Sastre-López A, Linares-Fano B, Guerra-Ordoñez JR, Alonso-Rojo AC, Prieto-Velasco M. Análisis del tiempo de entrenamiento y su relación con los episodios de peritonitis. Enferm Nefrol. 2020 Ene-Mar;23(1):54-59

\section{Resumen}

Introducción: El entrenamiento es fundamental para garantizar el éxito de la Diálisis Peritoneal.

Objetivo: Valorar la relación entre el tiempo de aprendizaje y la aparición del primer episodio de peritonitis, comparando entre pacientes jóvenes y ancianos.

Material y Método: Estudio observacional, retrospectivo. Se incluyó a los pacientes entrenados entre el 1 enero 2014 a 31 diciembre 2018, en el Complejo Asistencial Universitario de León. Se estudiaron variables demográficas, nivel de autonomía, tiempo de entrenamiento, primera peritonitis y germen causante. El punto de corte para diferenciar entre jóvenes y ancianos fue 70 años. Los datos se obtuvieron de la base de datos existente en nuestra unidad.

Resultados: Se estudiaron 112 pacientes, 68\% hombres, edad media $62,68 \pm 15,69$ años, $40 \%$ mayores de 70 años, el $82 \%$ eran autónomos para realizar la diálisis. La media de horas de entrenamiento fue 14,82 $\pm 6,55$; ancianos 18,13 $\pm 7,93$ horas, jóvenes $12,73 \pm 4,27,(p=0,004)$. El $25 \%$ tuvieron peritonitis, $64 \%$ por gérmenes Gram+. La media al primer episodio fue $266 \pm 283$ días. Los ancianos tardan 84 días menos de media en tener peritonitis.

\section{Correspondencia:}

Ana Isabel Aguilera Flórez E-mail: aaguilera@saludcastillayleon.es
Conclusiones: Aunque aparecieron más peritonitis en los pacientes que necesitaron más horas de entrenamiento, no se pudo establecer relación estadística alguna entre ambas variables; si bien los ancianos necesitan más tiempo para completar el entrenamiento y el primer episodio de peritonitis aparece más precozmente.

PALABRAS CLAVE: diálisis Peritoneal; aprendizaje; enseñanza; ancianos; peritonitis.

\section{Analysis of training time and its relationship with episodes of peritonitis}

\section{Abstract}

Introduction: Training is essential to ensure the success of peritoneal dialysis.

Objective: Assess the relationship between learning time and the appearance of the first episode of peritonitis, comparing between young and older patients.

Material and Method: Observational, retrospective study. Trained patients were included between January 1, 2014 and December 31, 2018, in the University Assistance Complex of León. Demographic variables, level of autonomy, training time, first peritonitis and causative germ were studied. The cut-off point to differentiate between young and older was 70 years. The data was obtained from the unit's databases. 
Results: 112 patients were studied, 68\% men, mean age $62.68 \pm 15.69$ years, $40 \%$ over 70 years, $82 \%$ were autonomous to perform dialysis. The average training hours were $14.82 \pm 6.55$; older $18.13 \pm 7.93$ hours, younger $12.73 \pm 4.27,(p=0.004) .25 \%$ had peritonitis, $64 \%$ for Gram+ germs. The mean appearance of the first episode was $266 \pm 283$ days. It takes 84 days less for the older patients to have peritonitis.

Conclusions: Although more peritonitis appeared in patients who needed more training hours, no significant differences were found. Older patients need more time to complete the training and the first episode of peritonitis appears earlier.

KEYWORDS: peritoneal dialysis; learning; teaching; elderly; peritonitis.

\section{Introducción}

En un programa de diálisis peritoneal (DP), el entrenamiento del paciente es un factor esencial y fundamental para garantizar el éxito de la técnica ${ }^{1}$.

Su objetivo es dotar al paciente y/o cuidador, de conocimientos y habilidades, que le permitan realizar el tratamiento en su domicilio de forma segura, autónoma y con las máximas garantías².

Se recomienda, establecer un plan de enseñanza progresivo que facilite, tanto el aprendizaje de conceptos, como la puesta en práctica de habilidades motoras específicas, necesarias para la realización de la técnica ${ }^{1}$.

Uno de los aspectos más importantes antes de comenzar la formación, es realizar una valoración integral de la persona que aprende, que abarque las diferentes esferas de la persona: clínica, funcional, mental y social permitiendo detectar los problemas, necesidades y capacidades, así como el apoyo familiar y las barreras para el aprendizaje 3 .

Otro de los aspectos a tener en cuenta, es la edad. Se estima, que las personas de edad avanzada son el grupo de pacientes en diálisis con mayor crecimiento en el mundo. En España, según el registro de diálisis y trasplante de la Sociedad Española de Nefrología de $2017^{4}$, el $59 \%$ de los pacientes incidentes tenían más de 65 años. El mayor número de patologías comórbidas, mayor riesgo de disfunción cognitiva y mayores niveles de fragilidad, son motivos por los que se cuestiona la idoneidad de la DP en las personas mayores, en ausencia de apoyo ${ }^{5}$.

La educación del paciente es esencial para lograr el autocuidado, permitirle mantener un buen estado de salud y prevenir complicaciones ${ }^{6}$.

Una de las complicaciones más frecuente y grave es la peritonitis, que puede impactar en la calidad de vida del paciente, siendo causa directa de morbimortalidad y fracaso de la técnica ${ }^{7}$. Dentro de las estrategias de prevención de peritonitis, uno de los puntos a destacar, son los programas de entrenamiento. Se recomienda que el entrenamiento sea realizado por personal de enfermería con las cualificaciones y experiencia apropiadas $^{7}$ y existe evidencia científica sobre la influencia que el método de entrenamiento tiene sobre el riesgo de peritonitis ${ }^{8}$, sin embargo, no existe un consenso sobre el tiempo que debe durar la capacitación o el momento y lugar ideal para realizarlo6.

El objetivo que nos hemos planteado en este estudio fue valorar el efecto del tiempo de entrenamiento sobre el primer episodio de peritonitis, comparando entre pacientes jóvenes y ancianos.

\section{Material y Método}

Se realizó un estudio observacional, retrospectivo, en la Unidad de DP del Complejo Asistencial Universitario de León, siendo el periodo de estudio desde el 1 de enero de 2014 al 31 de diciembre de 2018. Los criterios de inclusión fueron: pacientes adultos con enfermedad renal crónica avanzada (ERCA) entrenados para realizar cualquiera de las dos modalidades de $D P$, diálisis peritoneal continua ambulatoria (DPCA) o diálisis peritoneal automática (DPA). Los criterios de exclusión fueron: no haber concluido el entrenamiento y/o no iniciar el tratamiento en el domicilio. Las variables estudiadas fueron demográficas y clínicas, como: edad, sexo, modalidad dialítica, tiempo de entrenamiento, nivel de autonomía, tiempo a la primera peritonitis y germen causante, tiempo en diálisis peritoneal y causa de salida. Se consideró una edad de 70 años como punto de corte para diferenciar entre personas jóvenes y ancianas.

El entrenamiento se llevó a cabo en el hospital, de forma individualizada, por su enfermera referente; realizándose en días consecutivos, excepto el fin de semana. 
Cada día se realizaron 2 ó 3 sesiones de unos 45 minutos de duración.

En cuanto al nivel de autonomía se valoraron dos ítems, autónomos y asistidos. Se consideró asistido, todo paciente que necesitaba ayuda total o parcial para realizar la técnica. Los cambios de modalidad dialítica, de DPCA a DPA, se consideraron como nuevo entrenamiento.

Se realizó una hoja tipo Excel para la recogida de datos y se empleó el programa SPSS 15 para el tratamiento estadístico de los datos. Se calcularon las medidas de tendencia central y dispersión en las variables cuantitativas, expresando la media y desviación estándar. En las variables cualitativas se calcularon las frecuencias absolutas y los porcentajes. Se empleó la prueba $\mathrm{Chi}^{2}$ para la comparación de las variables cualitativas, el coeficiente de correlación de Pearson para establecer la asociación lineal entre variables cuantitativas, y la prueba T de Student para la comparación de medias, considerando significativa una $p<0,05$.

Los datos se obtuvieron de la base de datos y del registro de actividad de enfermería existente en nuestra unidad. No se han incluido datos de carácter personal que pudieran identificar a los pacientes de forma directa 0 indirecta, respetando los principios éticos y universales, así como, las normas internacionales de protección de datos y la legislación española vigente.

\section{Resultados}

La muestra fue de 112 pacientes, se excluyeron 3 pacientes que no iniciaron tratamiento en domicilio por disfunción del catéter. El $68 \%$ fueron hombres y el $32 \%$ mujeres, con una edad media de $62,68 \pm 15,69$ años. El 59,8\% tenían menos de 70 años y el 40,2\% eran mayores de 70 años. Los datos generales se presentan en la Tabla $\mathbf{l}$.

La media de tiempo de entrenamiento fue $14,90 \pm 6,80$ horas, repartido en $19,87 \pm 9,07$ sesiones durante $9,93 \pm 4,36$ días.

La asociación entre la duración del entrenamiento y el tiempo de aparición del primer episodio de peritonitis fue de $r=-0,19$, sin significación estadística (ver Figura 1).

Las horas de entrenamiento de los pacientes que tuvieron peritonitis fue de $14,78 \pm 6,8$ horas, y de $14,94 \pm 6,5$ horas en los no tuvieron ningún episodio, no presentando diferencia estadísticamente significativa $(p=0,9)$.
Tabla 1. Características generales de la muestra.

\begin{tabular}{lll}
\hline $\mathbf{n}$ & \multicolumn{1}{c}{112} \\
\hline Edad media (años) & \multicolumn{1}{c}{$62,68 \pm 15,69$} \\
\hline Sexo (Hombres/mujeres) & \multicolumn{1}{c}{$67,85 \%(76) / 32,15 \%(36)$} \\
\hline Modalidad DP & DPCA & $75 \%$ \\
& DPA & $25 \%$ \\
\hline Nivel de autonomía & Autónomos & $82 \%$ \\
& Asistidos & $18 \%$ \\
\hline \multirow{2}{*}{ Salidas } & Hemodiálisis & $25 \%$ \\
& Trasplante & $44,11 \%$ \\
& Exitus & $29,41 \%$ \\
& Recupera FR & $1,47 \%$ \\
\hline \multirow{2}{*}{ Permanencia en DP } & Promedio (meses) & $15,71 \pm 12,90$ \\
\hline
\end{tabular}

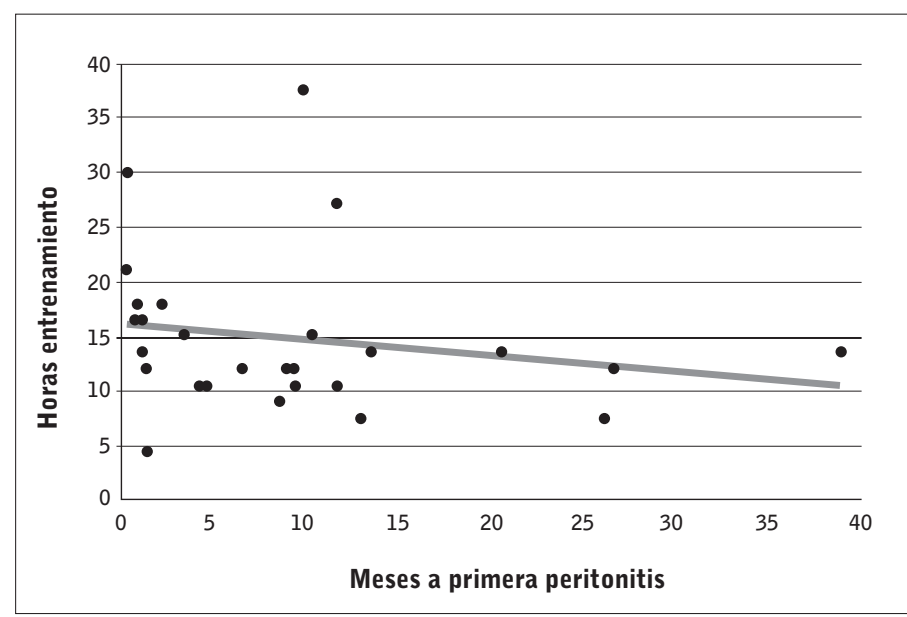

Figura 1. Correlación entre horas de entrenamiento y meses a primera peritonitis.

Los datos comparativos entre pacientes jóvenes y ancianos se muestran en la Tabla 2. Las personas mayores de 70 años, tardaron de media en aprender $18,13 \pm 7,93$ horas, frente a $12,73 \pm 4,27$ de los jóvenes, existiendo una diferencia estadísticamente significativa entre ambos $(p=0,004)$.

En los pacientes que tuvieron peritonitis, la media de días de exposición al primer episodio fue de $266 \pm 283$. Las personas mayores de 70 años, tardaron 84 días menos de media en tener un primer episodio, 226,06 $\pm 185,5$ días frente a $310,76 \pm 369,37$ días, $(p=0,062)$. La tasa global de peritonitis fue de 0,25 peritonitis/paciente-año. En cuanto a la peritonitis por modalidad de tratamiento, $73,7 \% \quad(n=22)$ estaban en DPCA y $25,4 \%(n=6)$ en DPA, aunque hay mayor numero de peritonitis en DPCA, no presentan diferencia estadísticamente significativa. 
Tabla 2. Comparación entre pacientes jóvenes y ancianos.

\begin{tabular}{|c|c|c|c|c|}
\hline & & $<70$ años & $\geq 70$ años & Valor $P(>0,05)$ \\
\hline n & & 67 & 45 & \\
\hline Edad Media (años) & & $52,77 \pm 15,68$ & $77,64 \pm 13,71$ & \\
\hline \multirow[t]{2}{*}{ Entrenamiento } & Promedio Horas & $12,73 \pm 4,27$ & $18,13 \pm 7,93$ & 0,004 \\
\hline & Promedio Días & $8,49 \pm 2,85$ & $12,08 \pm 5,2$ & 0,004 \\
\hline \multirow[t]{2}{*}{ Peritonitis } & $\mathrm{N}^{0}$ de episodios & 13 & 15 & \\
\hline & Días ler episodio (días) & $310,76 \pm 369,37$ & $226,06 \pm 185,5$ & 0,062 (N.S) \\
\hline Permanencia en DP & Promedio (meses) & $14,64 \pm 12,92$ & $17,6 \% \pm 13,22$ & 0,33 (N.S.) \\
\hline
\end{tabular}

* N.S.= No significativo

El tiempo de entrenamiento y la aparición del primer episodio de peritonitis, por modalidad dialítica, tampoco tuvo significancia estadística.

El $75 \%(n=84)$ de los pacientes estuvieron libres de peritonitis durante el tiempo que permanecieron en DP o durante el tiempo de estudio y el $25 \% \quad(n=28)$ presentaron un episodio.

Los microrganismos causantes de la infección fueron principalmente Gram positivos, destacando por su frecuencia, los Estafilococos Epidermidis y Aureus. Los datos se recogen en la Figura 2.

\section{Discusión}

La duración del entrenamiento es un parámetro que muestra una gran variabilidad, en una encuesta realizada a nivel mundial por la "International Society for Peritoneal Dialysis" (ISPD), se informaba de tiempos de aprendizaje que oscilaban de 6 a 96 horas 9 .

$n=28$

\section{Gram Positivos}

4 STF Epidermidis 4 STF Aureus

3 Corynebacterium

2 Enterococcus Faecalis

1 Enterococcus Faecium

1 Streptococo Mitis

3 Streptococo Salivarius

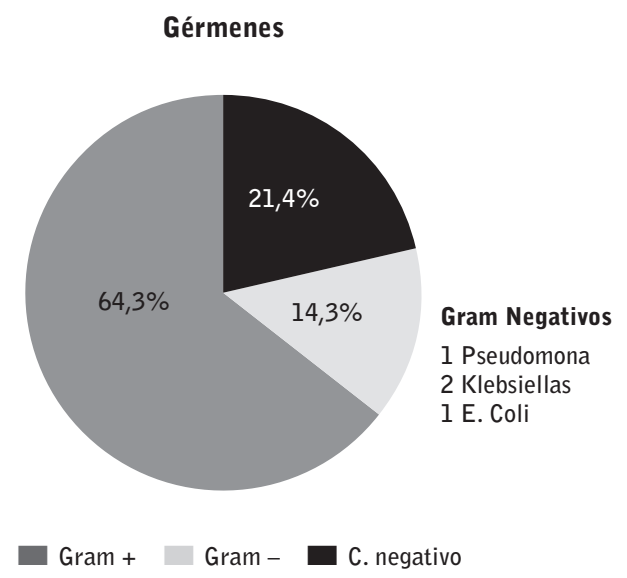

Figura 2. Gérmenes causantes del primer episodio de peritonitis. $\mathbf{S T F}=$ Estafilococo $\mathrm{E}$. Coli= Escherichia coli.
En nuestro estudio, hemos podido observar que, el tiempo de entrenamiento de los pacientes atendidos en nuestra unidad, es de unas 15 horas por paciente, dedicando alrededor de una hora y media por día. Estos resultados están en consonancia con los obtenidos en otros dos estudios: uno que informó de tiempos de entrenamiento de 15 horas con una dedicación de 1,1 hora diaria ${ }^{6}$ y otro que dedicaba 2 horas diarias durante 7 días $^{10}$.

Otro detalle observado, es que el tiempo de aprendizaje aumenta en las personas mayores de 70 años, que tardan alrededor de 5 horas más en aprender que los jóvenes. No resulta extraño, ya que con la edad, las capacidades cognitivas, funcionales y sensoriales pueden estar alteradas ${ }^{11}$.

La capacidad de aprendizaje de los pacientes varía, por tanto, es necesario emplear métodos de entrenamiento flexibles e individuales, respetando el ritmo y el estilo de aprendizaje propio de cada persona ${ }^{3,12}$. Sin embargo, y como apunta la ISPD, lo importante no es la duración del entrenamiento, sino conseguir que el paciente aprenda y realice la técnica de forma segura ${ }^{1}$.

La incidencia de peritonitis en nuestro estudio está dentro de los estándares establecidos, tanto nacionales como internacionales. Aparece en uno de cada cuatro pacientes y aunque es muy variable, sucede con mayor frecuencia, en ancianos y dentro del primer año en diálisis.

Según demuestran estudios recientes, existe una asociación entre los patrones de entrenamiento y la incidencia de peritonitis ${ }^{1,13}$. Un estudio multicéntrico que analizó la asociación entre las características del entrenamiento y la incidencia de peritonitis, concluyó que entrenamientos con una duración inferior a 15 horas totales, se asocia- 
ba con peores tasas de peritonitis ${ }^{6}$, a diferencia de 10 encontrado en nuestros resultados, en donde un mayor tiempo de entrenamiento no se relaciona con una aparición más rápida del primer episodio de peritonitis.

El riesgo de peritonitis en pacientes de edad avanzada es un tema controvertido, mientras algunos autores, la consideran como un factor de riesgo asociado con el primer episodio ${ }^{14,15}$, otros consideran que actualmente no hay diferencias en el riesgo de peritonitis y fracaso de la técnica entre pacientes mayores y jóvenes en DP11,16.

En cuanto al tiempo de exposición, en aquellos pacientes que presentaron peritonitis, fue de unos 9 meses, cabe destacar, que los ancianos tienen antes su primer episodio. Hecho que también se observa en otros estudios, que concluyen que la edad avanzada, el sexo masculino, el bajo nivel educativo y la hipoalbuminemia se asociaron con el primer episodio de peritonitis ${ }^{14}$.

No hemos encontrado diferencias respecto a la modalidad de tratamiento, aunque la frecuencia de episodios es mayor en los pacientes que están en DPCA.

Un hallazgo de este estudio fue, que las mujeres presentaron menor número de peritonitis y el primer episodio aparecía alrededor de dos meses más tarde que en los hombres. La bibliografía muestra disparidad de opiniones, mientras algunos autores consideran el sexo femenino como un factor de riesgo de peritonitis, muchas de ellas relacionadas con bacterias gramnegativos ${ }^{17}$; otros no encuentran diferencia ${ }^{15}$, incluso alguno concluye que es el sexo masculino el que presenta mayor riesgo ${ }^{14}$. Este resultado podría ser debido al menor número de mujeres incluidas en el estudio, el mayor porcentaje de estas en DPA, incluso que puedan ser mas cuidadosas a la hora de realizar los intercambios.

Los gérmenes causantes más frecuentes, fueron Gram positivos, por lo que el reentrenamiento, puede ser fundamental para la prevención de errores que puedan ocasionar riesgos de infección 1,10,12,18,19.

Destacar que, el $40 \%$ de nuestros pacientes son ancianos y solo una tercera parte necesitan ayuda para realizar la diálisis, estando la tasa de peritonitis en un nivel muy respetable. Estos datos están en la línea de los obtenidos en otros estudios y que informan de porcentajes que oscilan entre el 15 y el $17 \%$ de pacientes ancianos en DP que precisan ayuda ${ }^{20,21}$.
Una limitación a este estudio puede ser haber incluido los aprendizajes que suponen un cambio de modalidad, de DPCA a DPA, ya que podría ocasionar una variación en la duración del entrenamiento, infravalorando el tiempo de aprendizaje al no ser un paciente nuevo. En este estudio, la primera causa de abandono de la técnica es el trasplante seguido del exitus, algo razonable, dado el alto porcentaje de pacientes ancianos tratados, un dato que no hemos estudiado y que puede constituir una debilidad de este estudio, es el porcentaje de muertes atribuibles a los episodios de peritonitis. En base a los resultados obtenidos podemos concluir, que aunque aparecieron más peritonitis en los pacientes que necesitaron más horas de entrenamiento, no se pudo establecer relación estadística alguna entre ambas variables; si bien los ancianos necesitan más tiempo para completar el entrenamiento y el primer episodio de peritonitis aparece más precozmente.

Recibido: 28-11-19

Revisado: 05-12-19

Modificado: 08-01-20

Aceptado: 15-01-20

\section{Bibliografía}

1. Bernardini J, Price V, Figueiredo A. Pautas/Recomendaciones de la ISPD. Capacitación de pacientes en Dialisis Peritoneal. Perit Dial Int 2006;26:62532.

2. Granado A, Ruiz C, Arrieta J. Adiestramiento del paciente que se incorpora a diálisis peritoneal: $\mathrm{Vi}$ sitas domiciliarias. Reentrenamiento. Nefrología. 2006;26(Supl 4):S57-66.

3. Hurst $H$, Figueiredo AE. The needs of older patients for peritoneal dialysis: Training and support at home. Perit Dial Int. 2015;35(6):625-9. 
4. Registro Español de Enfermos Renales 2017 [Internet]. [Consultado 28 jun 2019]. Disponible en: https://www.senefro.org/contents/webstructure/Informe_REER_2017.pdf.

5. Brown EA, Johansson L. Dialysis Options for End-Stage Renal Disease in Older People. Nephron Clin Pr. 2011;119(1):10-3.

6. Figueiredo $A E$, De Moraes TP, Bernardini J, Poli-De-Figueiredo CE, Barretti $\mathrm{P}$, Olandoski $\mathrm{M}$, et al. Impact of patient training patterns on peritonitis rates in a large national cohort study. Nephrol Dial Transplant. 2015;30(1):137-42.

7. Li PK-T, Szeto CC, Piraino B, de Arteaga J, Fan S, Figueiredo $A E$, et al. ISPD Peritonitis Recommendations: 2016 Update on Prevention and Treatment. Perit Dial Int. 2016;36(5):481-508.

8. Piraino $B$, Bailie GR, Bernardini J, Boeschoten $E$, Gupta A, Holmes C, et al. Pautas/recomendaciones de la ispd recomendaciones para las infecciones relacionadas con diálisis peritoneal: actualización 2005. Perit Dial Int 2005;25(2):107-31.

9. Bernardini J, Price V, Figueiredo A, Riemann A, Leung $D$. International survey of peritoneal dialysis training programs. Perit Dial Int. 2006;6:658-63.

10. Bordin G, Casati M, Sicolo N, Zuccherato N, Eduati V. Patient Education in Peritoneal Dialysis: an Observational Study in Italy. J Ren Care. 2007;33(4):165-71.

11. Segall L, Nistor I, Van Biesen W, Brown EA, Heaf $J G$, Lindley $E$, et al. Dialysis modality choice in elderly patients with end-stage renal disease: a narrative review of the available evidence. Nephrol Dial Transplant. 2017;32(1):41-9.

12. Zhang L, Hawley CM, Johnson DW. Focus on peritoneal dialysis training: Working to decrease peritonitis rates. Nephrol Dial Transplant. 2016; $31(2): 214-22$
13. Campbell DJ, Johnson DW, Mudge DW, Gallagher MP, Craig JC. Prevention of peritoneal dialysis-related infections. Nephrol Dial Transpl. 2015;30:1461-72.

14. Fan $X$, Huang $R$, Wang J, Ye H, Guo Q, Yi C, et al. Risk factors for the first episode of peritonitis in Southern Chinese continuous ambulatory peritoneal dialysis patients. PLoS One. 2014;9(9):e107485.

15. Portolés J, Janeiro $D$, Lou-Arnal LM, López-Sánchez $P$, Ortega $M$, del Peso $G$ et al. Primer episodio de infección peritoneal: descripción y factores pronósticos. Nefrología. 2013;33(3):316-24.

16. Lim WH, Dogra GK, McDonald SP, Brown FG, Johnson DW. Compared with younger peritoneal dialysis patients, elderly patients have similar peritonitis-free survival and lower risk of technique failure, but higher risk of peritonitis-related mortality. Perit Dial Int. 2011;31(6):663-71.

17. Johnson D, Cho Y, Mehrotra R. Is female sex really a risk factor for infectious death in peritoneal dialysis? Perit Dial Int. 2013;33(5):475-8.

18. Szeto CC. Peritoneal dialysis-related infection in the older population. Perit Dial Int. 2015;35(6):65962.

19. Dong J, Chen Y. Impact of the bag exchange procedure on risk of peritonitis. Perit Dial Int. 2010;30(4):440-7.

20. Li PK-T, Law MC, Chow KM, Leung C-B, Kwan BC$H$, Chung $K Y$, et al. Good patient and technique survival in elderly patients on continuous ambulatory peritoneal dialysis. Perit Dial Int. 2007;27(2):196201.

21. Lim WH, Dogra GK, McDonald SP, Brown FG, Johnson DW. Compared with younger peritoneal dialysis patients, elderly patients have similar peritonitis-free survival and lower risk of technique failure, but higher risk of peritonitis-related mortality. Perit Dial Int. 2011;31(6):663-71.

Este artículo se distribuye bajo una Licencia Creative Commons Atribución-NoComercial 4.0 Internacional. https://creativecommons.org/licenses/by-nc/4.0/

Open Access (c) () 